\title{
En bloc Resection of Solitary Cranial Tumors: An Algorithmic Reconstructive Approach
}

\author{
Sabine A. Egeler, MD ${ }^{1}$ Anna Rose Johnson, $\mathrm{MPH}^{1} \quad$ Winona Wu, BA ${ }^{1} \quad$ Alexandra Bucknor, MBBS, MSc ${ }^{1}$ \\ Yen-Chou Chen, MD ${ }^{2}$ Ahmed B. Bayoumi, MD ${ }^{3}$ Ekkehard M. Kasper, MD, PhD ${ }^{2}$ \\ ${ }^{1}$ Division of Plastic Surgery, Beth Israel Deaconess Medical Center, \\ Harvard Medical School, Boston, Massachusetts \\ 2 Division of Neurosurgery, Hamilton General Hospital, Michael G. \\ DeGroote School of Medicine, McMaster University, Hamilton, Ontario \\ ${ }^{3}$ Department of Neurosurgery, Bahcesehir University, Bahcesehir, Turkey \\ Address for correspondence Ekkehard M. Kasper, MD, PhD, FAANS, \\ Division of Neurosurgery, Hamilton General Hospital, 237 Barton \\ Street East Hamilton, ON L8L 2X2, Canada \\ (e-mail: kaspere@mcmaster.ca).
}

J Reconstr Microsurg Open 2019;4:e14-e23.

\begin{abstract}
Background This study analyzes the surgical outcomes for single setting surgeries involving en-bloc solitary calvarial tumor resection in combination with three-layered reconstruction, presenting a novel planning algorithm.

Methods Data were retrieved for all patients undergoing single-stage tumor excision, using our novel three-layered reconstructive approach (duraplasty, cranioplasty, and soft tissue reconstruction) between 2005 and 2017 at a single tertiary hospital center. Patients $\geq 18$ years with a Karnofsky Performance score (KPS) $>70$ and a life expectancy of $>2$ months were included. Patient characteristics, surgical specifics, histological diagnoses, outcomes, and complications were reviewed.

Results Eighteen single-staged excisions and three-layered reconstructions were performed. Seven patients presented with primary tumors and 11 patients with metastases. Mean age was 62 years. Mean follow-up time was 39 months. Primary closure was used in 12 of 18 patients, microvascular free flap with skin grafting in 4 of 18 , and local advancement or rotational flap in 2 of 18 . Two compromised free flaps were revised. There was no flap necrosis, skin graft failure, or wound infection observed in this series. Neurosurgical complications included two cases with seizures, one sublesional intraparenchymal hematoma, one adjacent parenchymal infarct, one case of delayed postradiation cerebrospinal fluid leakage, and one case of subdural hemorrhage.

\section{Keywords}

- tumor

- cranioplasty

- scalp reconstruction

Conclusion En-bloc excision followed by three-layered reconstruction is a feasible and often suitable single-stage technique for complex solitary metastasis or primary calvarial tumors, which historically have been challenging to treat. It can offer an alternative approach to primary and metastatic calvarial tumors other than palliative treatment or hospice care.
\end{abstract}

The management of patients presenting with complex, solitary cranial tumors involving multiple compartments poses a technical challenge. The osseous calvarium is a frequent site of metastasis for systemic neoplasms, including cancer of the breast, lung, prostate, kidney, and thyroid. ${ }^{1-3}$

received

March 5, 2018

accepted after revision

November 27, 2018
DOI https://doi.org/

10.1055/s-0039-1678703.

ISSN 2377-0813.
Primary bone tumors of the skull comprise only 0.8 to $1 \%$ of all bone tumors. ${ }^{4}$ Indications for surgical resection of calvarial tumors have been poorly defined. Solitary metastasis, neurological deficit, widespread destruction of bone and dura, infiltration of the overlying scalp and skin,
Copyright $\odot 2019$ by Thieme Medical Publishers, Inc., 333 Seventh Avenue, New York, NY 10001, USA. Tel: +1(212) 584-4662.
License terms

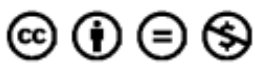


significant pain, and/or a corroborating pathological diagnosis are/is needed. ${ }^{2}$

En-bloc resection has been described as superior compared with other piecemeal or debulking procedures, as it is associated with achieving clear margins with minimal procedural tumor spillage. ${ }^{5,6}$ This treatment involves circumferential osteotomies to remove involved bone, affected adjacent layers and leaves substantial soft tissue defects requiring multistaged closure. ${ }^{6,7}$

Soft tissue closure in this region is challenging due to the inelastic skin and fibrous structure of the galea aponeurotica, which limits local skin mobility. ${ }^{8,9}$ Tissue expansion is an alternative for scalp tissue defect closure, replacing "like with like tissue."10 However, this approach requires multiple procedures prior to definitive surgical reconstruction and carries the risk of infection and extrusion. ${ }^{11}$

Factors complicating these reconstructions include a history of radiation therapy, preexisting scar formation, underlying compromise of the immune system (secondary to the use of steroids), and prior surgical intervention. ${ }^{4}$

This article reports our experience with complex, solitary calvarial tumors involving en bloc resection followed by "duraplasty, cranioplasty, and soft tissue" reconstruction. We developed a novel algorithm for a "three-layered" closure technique for complex lesions with dural, skull, and scalp involvement, presenting two illustrative cases.

\section{Patients and Methods}

A retrospective analysis of the electronic medical records was performed on all patients older than 18 years with solitary cranial tumors who underwent en bloc resection and "three-layer reconstruction" at our academic tertiary care center from 2005 to 2017. Patients with a Karnofsky Performance score (KPS) $>70$ and a life expectancy of $>2$ months were eligible for inclusion. Complex reconstructions of the soft tissue and skull were undertaken with a combined reconstructive and neurosurgical approach. The study was approved by the Institutional Review Board Committee.

\section{Patient and Surgical Data}

Patient characteristics included: gender, age, race, comorbidities, medical management, and follow-up in months. Tumorspecific variables include: clinical presentation, tumor site and dimensions, pathological diagnosis, skull involvement, brain invasion, and cancer recurrence. Operative details were analyzed from the perspective of the respective specialty.

Neurosurgical specifics included: craniotomy, lobotomy, type of allograft or implant material, postoperative outcomes, complications, and the need for chemotherapy or adjuvant radiotherapy.

Reconstructive surgical information included: width of the defect, depth of defect, type of reconstruction, flap type, recipient vessel type, need for vein grafts, and necessity of skin graft.

\section{Three-Layer Reconstruction}

Three-layer reconstruction encompasses the simultaneous reconstruction of a multilayered cranial defect including scalp, bone, dura (and possibly brain). The resection of the brain-invading component of such lesions is not notably different from other resections in neurosurgery; there is no unique aspect of the resection to report.

The next step addresses the closure of the cerebrospinal fluid (CSF) containing compartment. Dural reconstruction is performed as the first layer using a biosynthetic dural allograft (Duraform, Raynham, MA), as by the preference of the neurosurgeon.

The second layer includes skull reconstruction, which is performed by using a custom-molded titanium mesh (Synthes, Zuchwil, Switzerland) enhanced with methylmethacrylate matrix (MM) soaked in antibiotic solution (Bacitracin). Alternatively, prefabricated antibiotic enriched MEDPOR allograft (Portage, MI) can also be used.

The allograft is implanted to the healthy surrounding bone extending ca. 0.5 inch beyond the cut out of the bone. Intraoperatively, the allograft can be individually cut to size and molded to follow the contour of the skull for a better cosmetic fit. With the use of osteosynthesis miniplates and screws (Synthes), the mesh is attached. After the mesh graft is secured, it is augmented with porous methacrylate paste creating it a firmer construct with higher mechanical strength, prior to skin closure.

The third layer includes soft tissue reconstruction of the scalp. Primary closure is indicated and feasible if the scalp defect over one hemiconvexity measures less than $3 \mathrm{~cm}$ in maximum width (-Fig. 1). Soft tissue defects measuring between 3 and $6 \mathrm{~cm}$ in width can be managed by a local scalp flap (e.g., rotational flap, V-Y advancement flaps, Orticochea

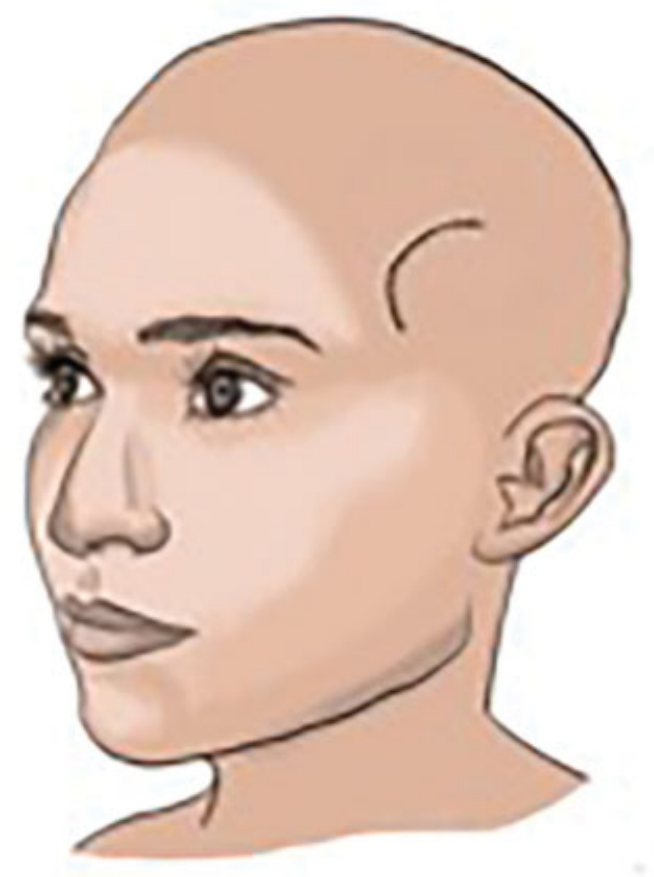

Fig. 1 Scalp defect less than $3 \mathrm{~cm}$ in maximum width. 


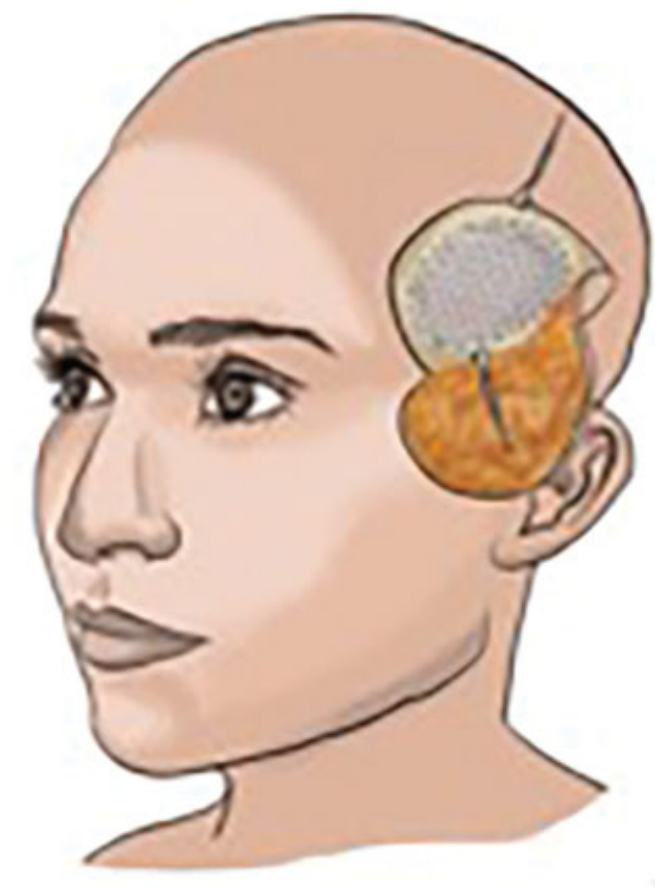

Fig. 2 Scalp defect between 3 and $6 \mathrm{~cm}$ in width.

flaps, Z-plasty) (-Fig. 2). ${ }^{12-14}$ Finally, we consider microvascular free flap reconstruction necessary when the remaining scalp defect measures $>6 \mathrm{~cm}$ in maximum diameter or the missing tissue area exceeds $36 \mathrm{~cm}^{2}$ (-Fig. 3). This three-layered approach is detailed in - Fig. 4.

\section{Results}

\section{Demographics}

Eighteen patients were included. Seven patients were male and 11 were female with a mean age of 62 years (range: 20 85 years). The patients were predominately Caucasian $(n=12)$. There were no active smokers or diabetic patients. Eight patients had underlying cardiovascular disease. All patients with malignant lesions had a preoperative KPS score above 70 , with controlled systemic disease.

Of the 18 cases, 7 presented with a protruding scalp mass and 4 presented with an open scalp defect with exposed calvarium or mesh. Additionally, three patients had symptoms of increased intracranial pressure. Two patients had additional focal neurological deficits. Two cases were discovered incidentally during routine follow-up for other preexisting conditions.

\section{Pathology}

Of all calvarial lesions, nine were of metastatic origin: five breast cancers, one bronchogenic non-small cell lung carcinoma, one prostate cancer, one pancreatic cancer, and one hepatocellular carcinoma. Four cases presented as primary

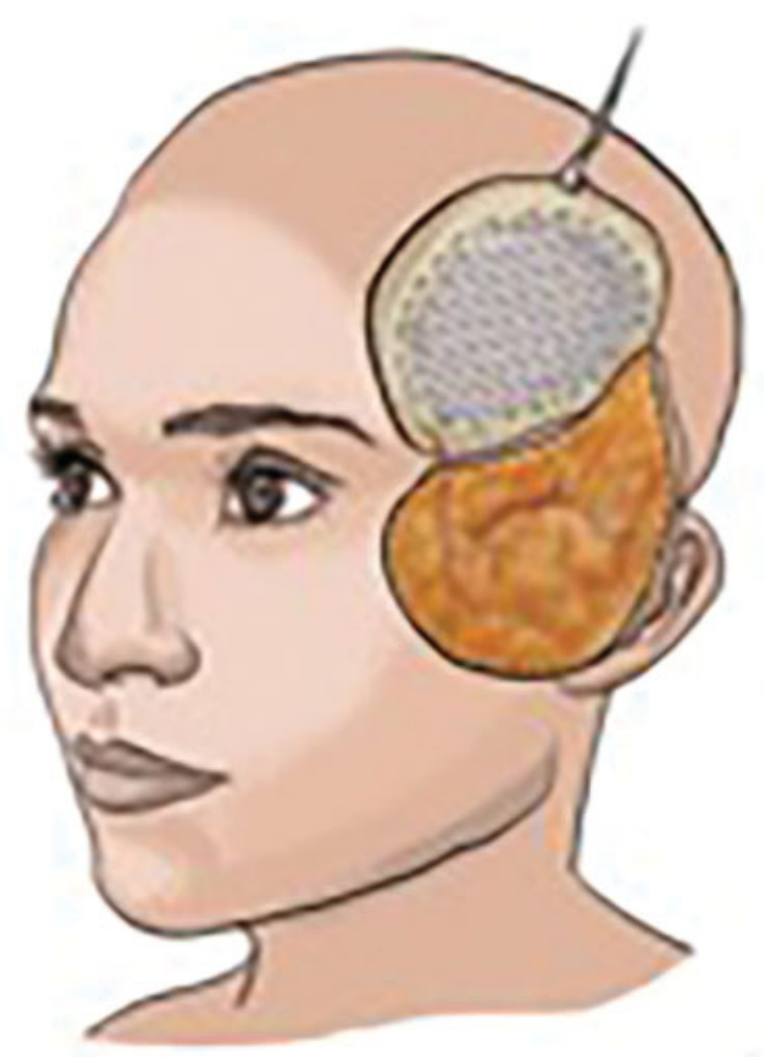

Fig. 3 Scalp defect $>6 \mathrm{~cm}$ in width.

malignant tumors (dermatofibrosarcoma protuberans [DFSP], sarcoma, squamous cell carcinoma, and anaplastic meningioma), one as an atypical meningioma (World Health Organization; WHO grade II) and three cases were benign cranial WHO I or II tumors (cavernous hemangioma, epidermoid, and hemangiopericytoma). One patient presented with a recurrent meningioma (WHO grade I).

Sixteen cases were located supratentorially and two cases involved the posterior fossa. Six cases showed evidence of transdural brain invasion requiring intraoperative microscopic dissection of tumor from the brain parenchyma. Two cases displayed deep extension and required microscopic brain dissection. These cases required soft tissue coverage for superficial extension. In two cases, the lesions were completely intraosseous with limited involvement of the adjacent layers.

Four cases (anaplastic meningioma, meningioma, metastatic carcinoma of the lung, and one from the liver) required focal postoperative radiation therapy, while two cases (metastatic lesion from the breast and from the pancreas) needed adjuvant whole brain radiation therapy following surgery (-Table $\mathbf{1}$ ).

\section{Reconstructive Management}

Dural reconstruction was performed using biosynthetic allograft duraplasty (Duraform). A titanium mesh (Synthes) was used for 16 patients combined with MM for skull reconstruction. One patient did not require MM. Additionally, one patient who had a distinct intraosseous neoplasm with lymphangiomatosis 


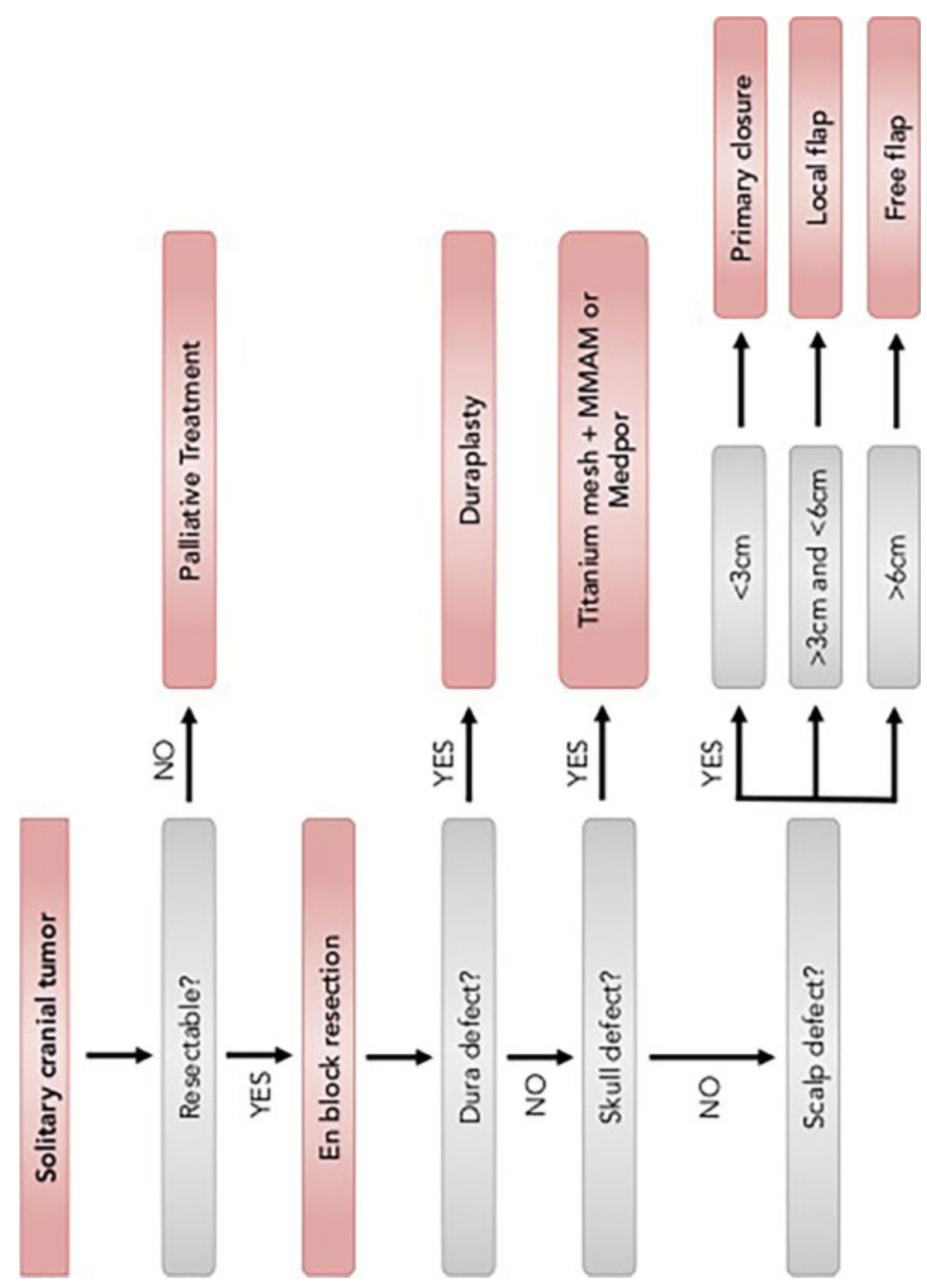

Fig. 4 Three-layered approach.

with a visible infiltrated and destructed bone on a densitygraded computed tomography (CT) scan received a prefabricated medpor allograft. Prediction of craniectomy size was performed on the presurgical CT scan. Virtual imaging and planning software were used to define the affected bone combined with $8 \mathrm{~mm}$ margin. A presurgical explant model was used. The actual craniectomy was performed by loading the same presurgical imaging set into an intraoperative image guidance system allowing demarcation and resection of the pathological bone segment. The prefabricated Medpor graft allowed us to close the newly created defect in a single step during the same surgery.

Twelve patients underwent primary closure for a defect measuring less than $3 \mathrm{~cm}$. One patient received a bilateral scalp advancement flap combined with an $8 \times 26 \mathrm{~cm}$ split- thickness skin graft (STSG). Another patient received a rotational flap with subgaleal release. Defects larger than $6 \mathrm{~cm}$ were reconstructed using free muscle flaps. The latissimus dorsi (LD) flap was used in two cases. The serratus anterior flap and the anterolateral thigh free flap were both used in one case (-Table 2 ). The superficial temporal artery was used as the recipient vessel in all cases.

\section{Postoperative Outcomes}

Mean postoperative hospital stay was 6.8 days. Follow-up periods ranged from 6 to 86 months. Five patients had neurosurgical postoperative complications. These included: one episode of intraparenchymal hematoma, one recurrent chronic subdural hematoma, one middle cerebral artery infarction, and one patient with increased frequency of seizures at 6 months. 


\begin{tabular}{|c|c|c|c|c|c|c|c|c|c|c|c|c|c|c|c|c|c|c|}
\hline 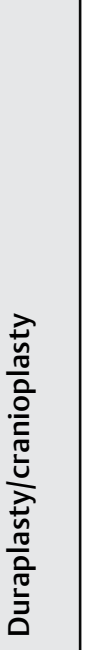 & 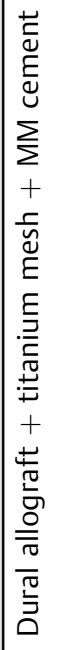 & 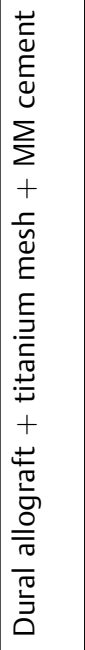 & 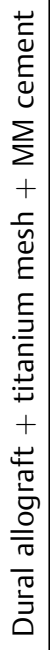 & 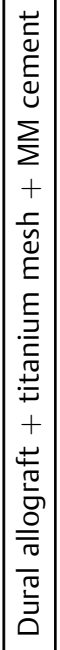 & 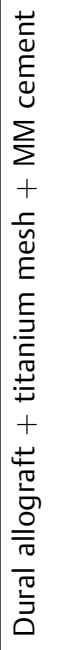 & 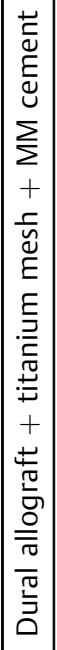 & 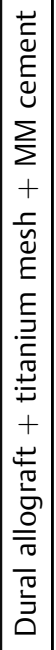 & 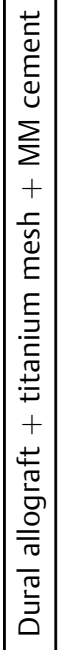 & 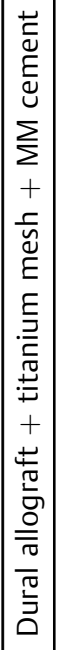 & 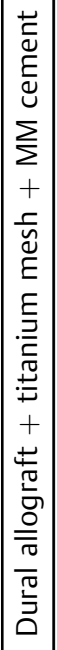 & 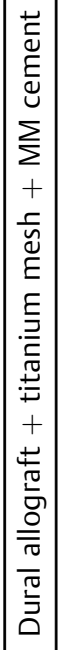 & 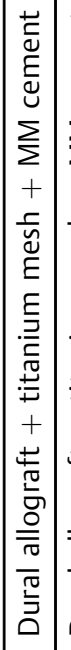 & 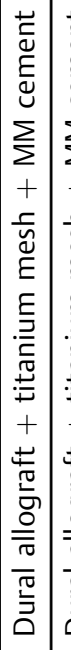 & 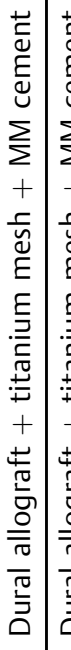 & 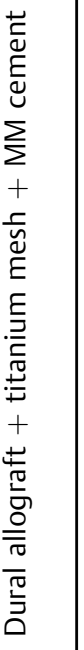 & 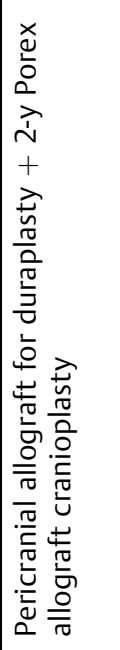 & 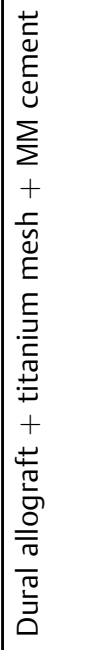 & 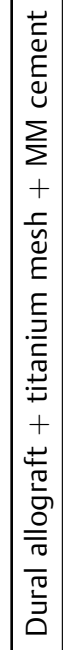 \\
\hline 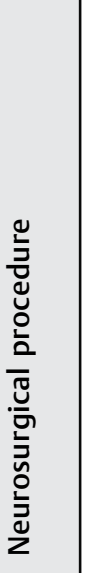 & 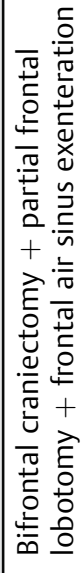 & 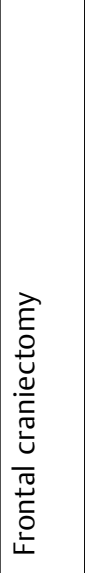 & 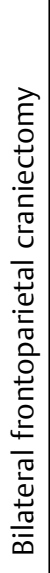 & 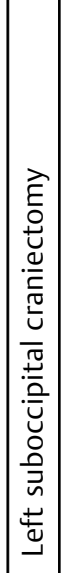 & 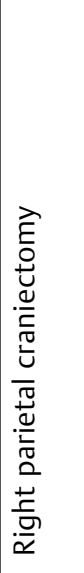 & 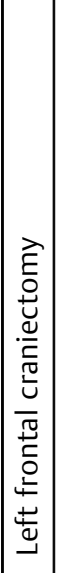 & 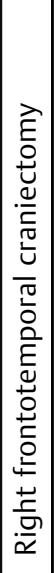 & 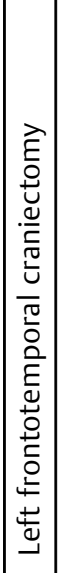 & 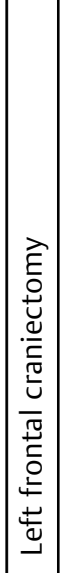 & 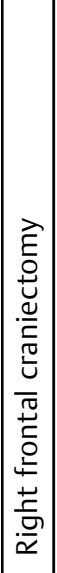 & 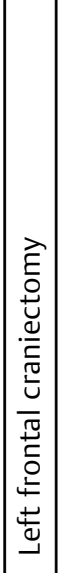 & 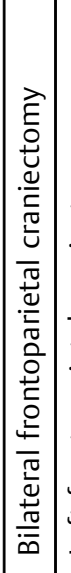 & 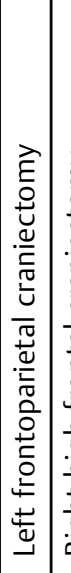 & 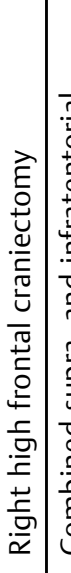 & 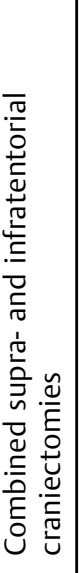 & 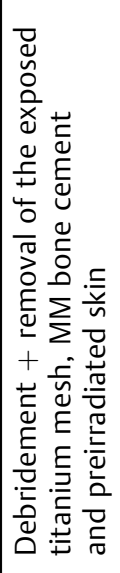 & 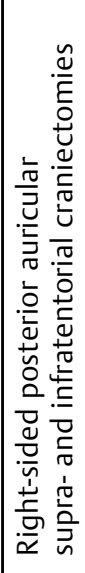 & 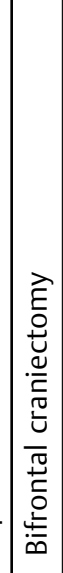 \\
\hline 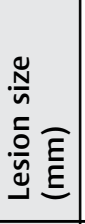 & 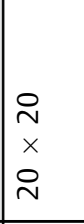 & $\begin{array}{l}\stackrel{\llcorner}{N} \\
\times \\
\stackrel{\Perp}{N}\end{array}$ & 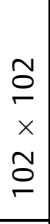 & $\stackrel{\curvearrowright}{\sim}$ & $\begin{array}{l}\stackrel{n}{n} \\
x \\
\stackrel{n}{N}\end{array}$ & $\begin{array}{l}1 \\
x \\
6\end{array}$ & $\begin{array}{c}\text { 오 } \\
\times \\
\text { 이 } \\
\end{array}$ & $\begin{array}{l}\bar{\sim} \\
\times \\
\tilde{m} \\
\end{array}$ & \begin{tabular}{|c|}
$\stackrel{n}{n}$ \\
$m$ \\
$\times$ \\
$\dot{q}$ \\
\end{tabular} & \begin{tabular}{|l|}
$x$ \\
0 \\
$\check{x}$ \\
$\times$ \\
$\stackrel{2}{N}$ \\
\end{tabular} & 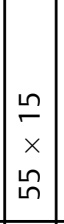 & 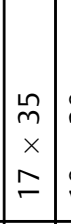 & $\begin{array}{l}\stackrel{0}{m} \\
\times \\
\circ \\
\end{array}$ & 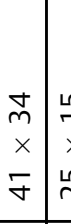 & $\begin{array}{l}\stackrel{n}{\infty} \\
\times \\
\stackrel{n}{\sim} \\
\end{array}$ & $\infty$ & 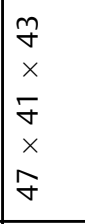 & $\begin{array}{l}\infty \\
\check{\infty} \\
\times \\
\pm \\
\times \\
\bar{\sim} \\
\end{array}$ \\
\hline 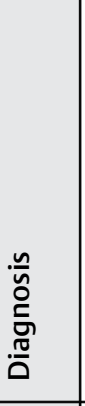 & 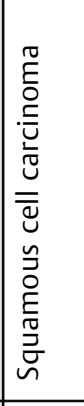 & 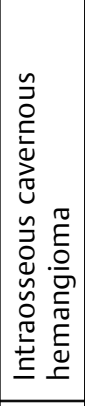 & $\begin{array}{l}0 \\
\tilde{\Xi} \\
0 \\
\tilde{U} \\
\tilde{n}\end{array}$ & 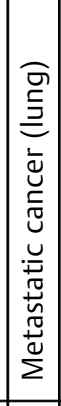 & 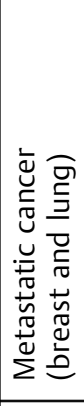 & 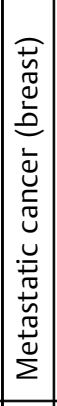 & 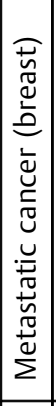 & 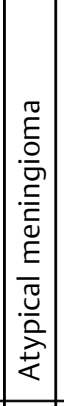 & \begin{tabular}{|l} 
जิ \\
苍
\end{tabular} & 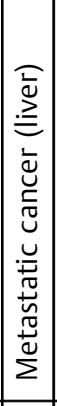 & 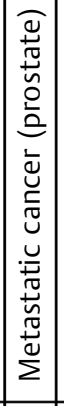 & 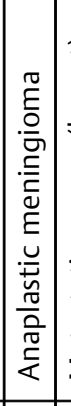 & 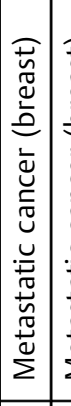 & 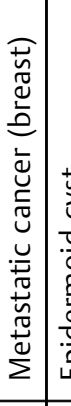 & 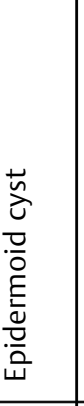 & 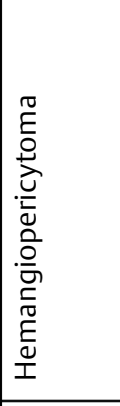 & 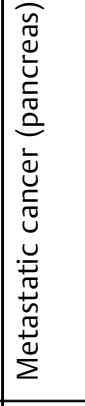 & 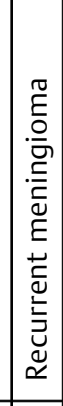 \\
\hline 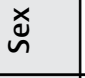 & 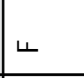 & $\Sigma$ & $\Sigma$ & 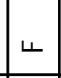 & \llcorner & $\angle$ & $\angle$ & 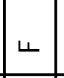 & $\Sigma$ & $\Sigma$ & $\Sigma$ & $\Sigma$ & $\leftarrow$ & \begin{tabular}{l|l} 
& 4 \\
\end{tabular} & \llcorner & 4 & 4 & $\Sigma$ \\
\hline 安 & $\stackrel{\llcorner}{\infty}$ & in & $\stackrel{\sim}{\sim}$ & $\curvearrowright$ & 6 & เి & $\curvearrowright$ & 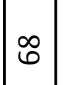 & $\stackrel{\sim}{\sim}$ & 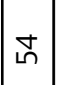 & 8 & $\stackrel{0}{r}$ & นู & $\stackrel{\infty}{\infty} \mid \supseteqq$ & $\stackrel{i}{N}$ & $\stackrel{9}{r}$ & in & 8 \\
\hline $\overrightarrow{\tilde{n}}$ & - & $\sim$ & $m$ & 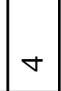 & in & 6 & 1 & $\infty$ & $a$ & -1 & $F$ & $\simeq$ & $\stackrel{m}{r}$ & \pm & 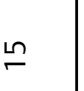 & - & 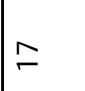 & $\stackrel{\infty}{\ulcorner}$ \\
\hline
\end{tabular}




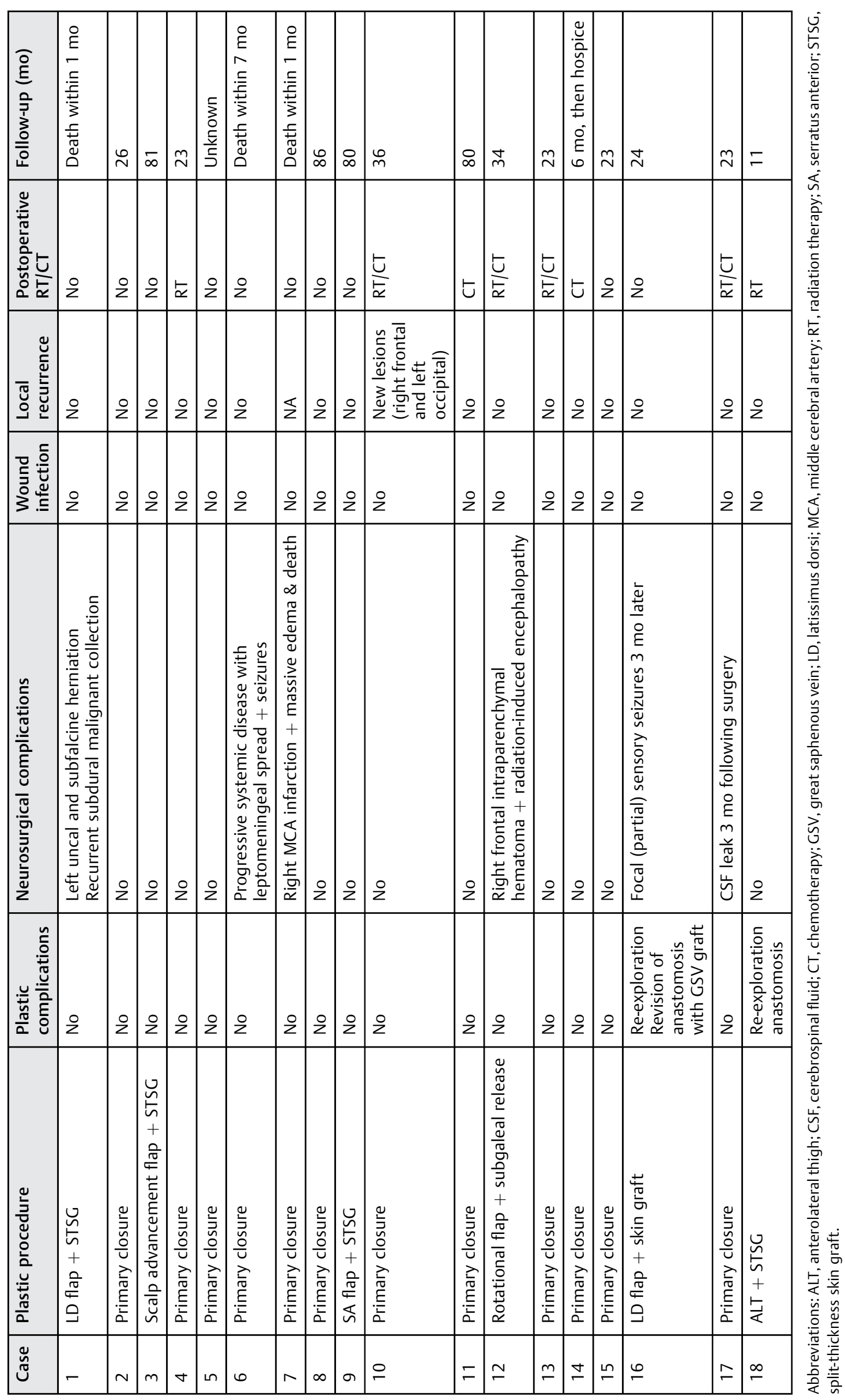


Two patients died within 1 month of surgery: one after right middle cerebral artery infarction and one due to recurrent subdural hemorrhage. In both of these cases, goals of care were changed and the families of both patients opted to withdraw active care.

Furthermore, 2 months postoperatively after having additional radiation therapy, a delayed complication of CSF leak was identified in one case, requiring neurosurgical intervention.

Two plastic surgery cases had a complication of a compromised free flap having the superficial temporal artery as the recipient vessel in both cases. One needed an anastomosis revision with the use of the great saphenous vein graft to restore blood flow. The other case needed hematoma and clot evacuation followed by anastomotic revision. Both free flaps were salvaged.

One episode of local disease recurrence was observed during the reported follow-up period. There was an additional case of regional malignancy recurrence of two skull lesions 16 months after the original resection. This was successfully treated with stereotactic radiosurgery. It should be noted that these lesions were not anatomically located in the resected region.

\section{Cases}

\section{Case Illustration (Case 9)}

A 24-year-old male patient presented to our clinic with a left frontoparietal scalp lesion. Medical history was significant for a cyst resection of the scalp 15 months prior to presentation at a hospital abroad. Physical examination revealed a $4.0 \times 3.5 \mathrm{~cm}$ firm, yellow-orange fungating mass that was centered over his prior surgical scalp incision. Head CT scan revealed a $2.1 \times 2.4 \mathrm{~cm}$ parietal lesion with periosteal infiltration and without parenchymal or meningeal involvement. Histopathology was consistent with DFSP.

A joint neurosurgery/plastic surgery team planned a single-stage en bloc resection and three-layered reconstruction. An image-guided technique was used to demarcate the intraosseous part of the skull lesion. The patient was positioned in the right lateral decubitus position with the head secured by contralateral Mayfield pins.

Soft tissue wide-margin resection was performed and pathological specimens were obtained. A high-frontal craniotomy followed by the complex en bloc resection was performed. After removing the tumor, dural defect closure was achieved using an onlay dural allograft.

A titanium mesh was molded to the skull defect, and miniscrews were used for circumferential fixation. Bacitracin-impregnated MM was applied to augment the titanium mesh to form a stable construct. A complex soft tissue defect measuring $12 \times 13 \mathrm{~cm}$ remained.

The serratus muscle free flap with its inferior four slips was harvested for soft tissue reconstruction. The superficial temporal artery and vein were identified as the recipient vessels. A STSG was harvested from the left lateral thigh for free flap coverage. One Jackson-Pratt drain and one Penrose drain were placed at the time of scalp closure. Xeroform was used for wound coverage and the patient was transferred to

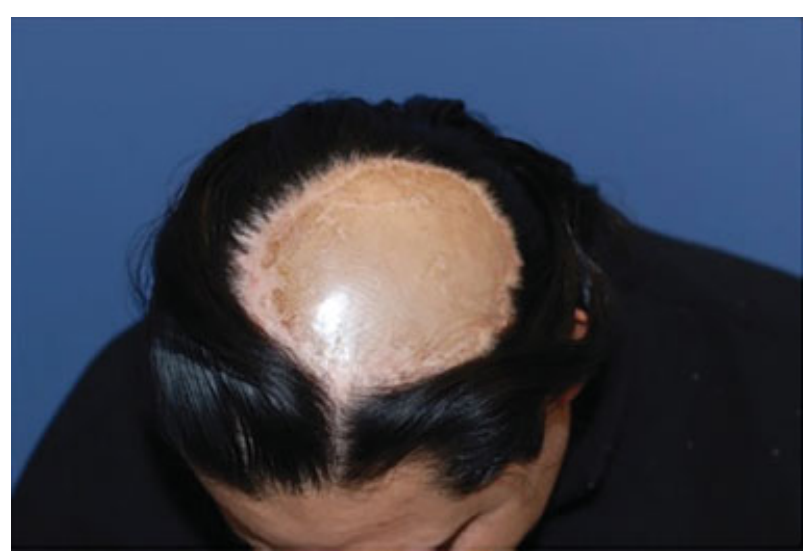

Fig. 5 The end result of case 9 .

the intensive care unit for postoperative management. Flap perfusion was monitored hourly using an external Doppler.

The patient had an unremarkable postoperative course with no cancer recurrence observed during a follow-up period of 5 years ( $\mathbf{- F i g}$. 5 ).

\section{Case Illustration (Case 3)}

A 74-year-old male presented to our clinic for surgical evaluation after excision of a soft tissue mass located in the high vertex of the scalp. The patient underwent Mohs micrographic surgery at another facility, which revealed a malignant spindle neoplasm with extension into the deep margins of the fascia specimens and likely bony infiltration. A scalp defect with bone exposure of $10.2 \times 10.2 \mathrm{~cm}$ was present on the upper scalp at the vertex.

Preoperative magnetic resonance imaging confirmed a large scalp soft tissue defect in the posterior vertex with signal abnormalities extending into the bone at the resection site. No parenchymal extension was present. A complex bilateral frontoparietal craniectomy was performed after intraoperative image guidance, confirming tumor extension.

Infiltrated bone was removed and $2 \mathrm{~cm}$ safety margins were confirmed. The wound was irrigated with bacitracin. Onlay dural allograft was used to repair the dural defect. A titanium mesh cranioplasty was molded to the skull contour and applied to the bony defect using Synthes' miniplating system for fixation, achieving solid and stabile construct. The mesh was filled with a bacitracin-impregnated methacrylate optimizing the contour of the replaced skull portion. Cranioplasty hardware was visible at the center of the defect site which measured $8 \mathrm{~cm}$ in diameter (-Fig. 6).

The reconstructive team chose bilateral opposing scalp advancement flaps for defect closure. The flaps were developed in a circumferential manner and extended laterally from the defect toward the anterior portion of the temporal line. The flaps were raised off the pericranium prior to medial advancement. They were sutured in place to cover the defect with the implanted cranioplasty. A skin graft measuring $8 \times 26 \mathrm{~cm}$ was harvested from the left thigh and used to cover the temporal scalp donor defect. Vacuum-assisted closure dressings were applied to the grafted area. 


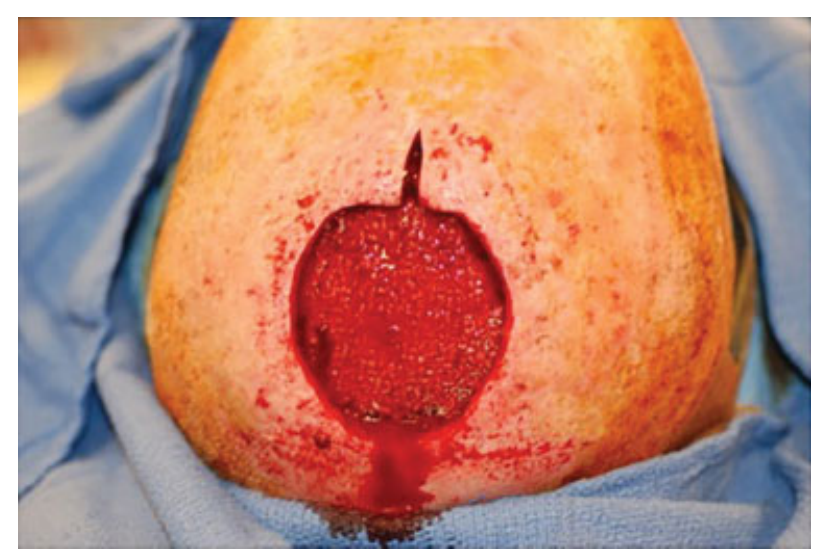

Fig. 6 Intraoperative defect of case 3 displaying the titanium mesh.

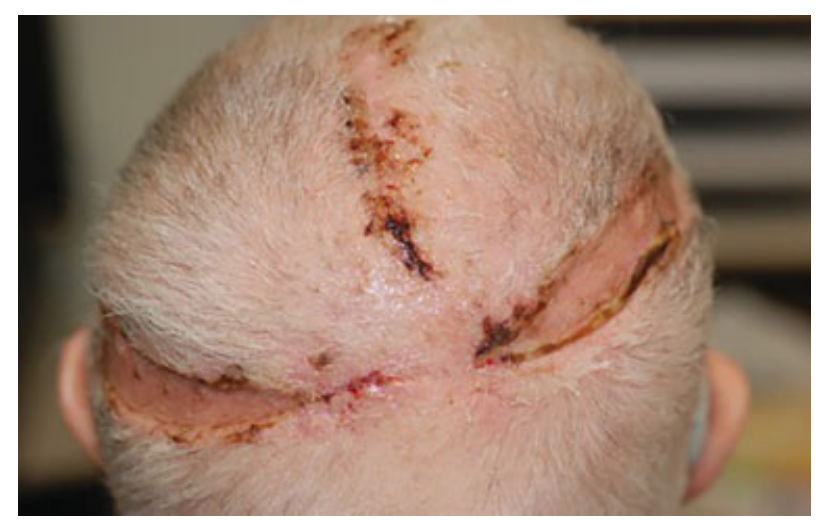

Fig. 7 Postoperative image.

The patient had an unremarkable postoperative course. There was no evidence of wound infection, flap necrosis, or skin graft failure (-Fig. 7). The patient has no evidence of tumor recurrence during a follow-up period of 6 years and has required no revision surgeries (-Fig. 8).

\section{Discussion}

In this case series, we present our novel algorithm for en bloc resection and three-layered reconstruction (dura, cranium, and scalp skin) of solitary calvarial tumors using an interdisciplinary approach.

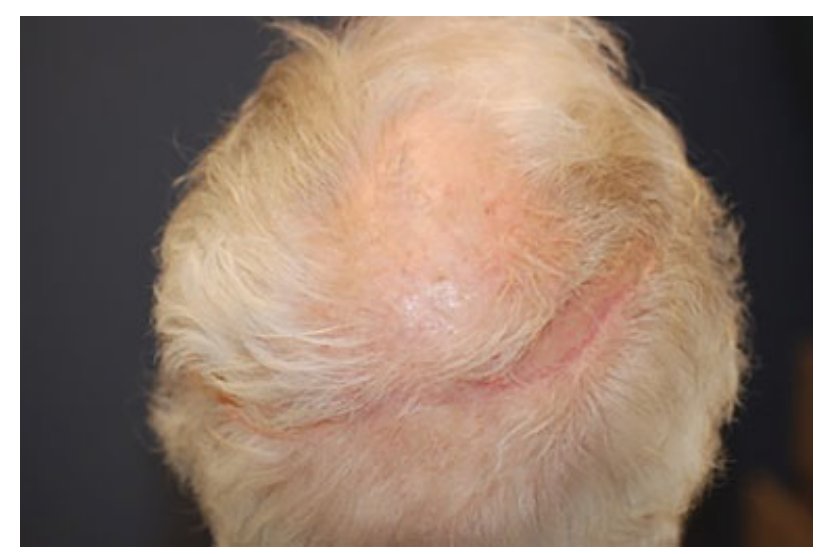

Fig. 8 The end result of case 3 .
En bloc resection of calvarial tumors is a complex procedure, requiring not only resection of the scalp skin and bone, but often requires resection of the dura. We used a biosynthetic allograft duraplasty (Duraform) in our study. Dura closure can be done through different types of sealant techniques and substitutes preventing CSF leakage, which occurs in 4 to $32 \%$ in all cranial surgeries. ${ }^{15}$ This complication has been correlated with meningitis, delayed wound healing, epidural infections, and pneumochephalus. ${ }^{16}$ A recent systematic review by Kinaci et al concluded no statistical significant difference in CSF leakage with the use of dural sealants compared with no sealants. ${ }^{16}$

Achieving tension-free closure in scalp reconstruction is of the utmost importance to enable preservation of the underlying alloplastic cranioplasty. These defects should be covered with well-vascularized local or free flaps. Closure under tension may lead to scar alopecia, dehiscence, and infection. ${ }^{17}$ These, in turn, can cause exposure of the hardware and result in eventual hardware loss. ${ }^{9}$

Due to the inelasticity of the scalp, only defects less than $3 \mathrm{~cm}$ in width should be closed primarily. Depending on the soft tissue quality, undermining the scalp or scoring of the galea may help reduce tension during wound closure. ${ }^{18}$ Although healing by secondary intention or skin grafting is indicated in instances of superficial tumor excision or trauma when the pericranium remains intact, ${ }^{19}$ it is not applicable to our case series due to the soft tissue reconstruction needed to cover the allograft implant. Additional considerations include a history of radiation therapy, which can lead to skin stiffening and inability to achieve primary closure with advancement flaps. ${ }^{9}$

Local flaps (mostly used in defects between 3 and $6 \mathrm{~cm}$ ) have the advantage of achieving defect coverage with hairbearing, well-vascularized skin. ${ }^{20}$ Different types of local flaps can be used, including advancement flaps, rotational flaps, or regional flaps. Several procedures such as undermining, galeal scoring, or a back cut may help reduce tension. ${ }^{18} \mathrm{~A}$ local flap is often combined with a STSG, as illustrated in our case series. This combination provides the opportunity to achieve extra length and tension-free closure of the skin. Scalp defects up to 6 to $8 \mathrm{~cm}$ in width can often be closed using local flaps. Doubleopposing flaps may be suitable for larger scalp defects, as seen in our case illustration. The Orticochea-type flap design has been reported to close large defects up to $15 \times 17 \mathrm{~cm}$ in size. ${ }^{21}$ However, we operate within more conservative parameters, setting an upper limit of $6 \mathrm{~cm}$ for local flap closure.

Our case series is mostly composed of metastatic cancers and primary malignant tumors, with neoadjuvant or postoperative radiotherapy. Our three-layered closure expands the indication of free flap reconstruction of scalp defects to those exceeding $6 \mathrm{~cm}$ in width, thus allowing patients who were previously considered inoperable to seek surgical care and not pursue palliation only.

In our case series, two patients with large scalp defects were reconstructed using a LD musculocutaneous flap. A free flap is a safe and reliable reconstruction approach that can cover larger complex scalp defects ( $>6 \mathrm{~cm}$ in width), especially in the context of aggressive malignant tumor resection. 
Among the free flap options, the LD musculocutaneous flap is considered the workhorse of the scalp reconstruction. ${ }^{22}$ This is due to its broad, thin, well-vascularized muscle and consistent axial vascular anatomy including length of vessel $(\sim 10 \mathrm{~cm})^{8,9,20,22}$ Furthermore, vascularized bone (rib or scapula) can be incorporated with the combined LD flap as a composite cranial defect reconstruction. ${ }^{23}$

The free serratus anterior muscle flap is another option for scalp defects. ${ }^{21}$ In our case the inferior four muscle slips were harvested. However, the serratus anterior muscle flap is usually limited to smaller scalp defects, as it has a surface area of $\sim 10 \times 12 \mathrm{~cm}$ compared with the $30 \times 11 \mathrm{~cm}$ of the LD musculocutaneous flap. ${ }^{24}$ In our case, we chose the serratus muscle free flap after the pedicle length of the LD flap was determined to be insufficient.

Moreover, an important technical challenge during a combined neuro- and plastic surgical approach is the patient positioning. The LD free flap combined with a STSG has the disadvantage of changing patient position during flap harvesting. ${ }^{25}$ However, we were able to avoid patient reposition during harvest of the serratus anterior flap due to the use of lateral decubitus positioning and contralateral Mayfield pins in the cranium.

With increasing use of flap-monitoring technology, our algorithm becomes easier to implement and all the more feasible. In the past, postoperative monitoring of free flaps has relied on frequent clinical examinations to verify flap perfusion; however, Doppler technology, including external and implantable devices, has allowed for reliable flap monitoring and associated decreased rates of flap failure. ${ }^{26,27}$ New techniques have further improved the postoperative monitoring process, including "ViOptix Tissue Oximeter device" using near-infrared spectroscopy and "SmartBandage" using oxygenation sensing paint-on bandage, to monitor tissue oxygenation. ${ }^{26,28}$

Potential limitations of this study are lack of a control group undergoing conservative therapy, coupled with its retrospective nature. This limits the ability to control for confounding factors or preexisting bias in patient selection. Moreover, the generalizability of the results may be limited by the small sample size. Moving forward, our case series establishes a fundamental foundation for utilization of the three-layer en bloc resection and reconstruction.

\section{Conclusion}

En-bloc excision followed by immediate three-layered reconstruction is a promising single-stage approach for solitary metastasis or primary calvarial tumors. It demonstrates the utility of an interdisciplinary neurosurgical and reconstructive approach that enables soft tissue reconstruction using different methods of soft tissue closure commensurate to defect size while minimizing postoperative complications and rate of tumor recurrence. Further studies are needed to document its utility in a larger population.

\section{Conflict of Interest}

None declared.

\section{References}

1 Bayoumi AB, Nigim F, Mahadevan A, et al. A case-series of solitary skull neoplastic lesions treated with en-bloc resection and complex cranioplasty. J Surg 2015;3(01):1-8

2 Lee EI, Chao AH, Skoracki RJ, Yu P, DeMonte F, Hanasono MM. Outcomes of calvarial reconstruction in cancer patients. Plast Reconstr Surg 2014;133(03):675-682

3 Stark AM. Neurosurgical treatment of breast cancer metastases to the neurocranium. Pathol Res Int 2010;2011:549847

4 Kakkar A, Nambirajan A, Suri V, et al. Primary bone tumors of the skull: spectrum of 125 cases, with review of literature. J Neurol Surg B Skull Base 2016;77(04):319-325

5 Cöloğlu H, Özkan B, Sener M, Uysal AÇ, Borman H. The management of non-melanocytic skin malignancies of the scalp and calvarium. Indian J Plast Surg 2014;47(01):36-42

6 Hendryk S, Czecior E, Misiołek M, Namysłowski G, Mrówka R. Surgical strategies in the removal of malignant tumors and benign lesions of the anterior skull base. Neurosurg Rev 2004;27(03): 205-213

7 Kasper EM, Ridgway EB, Rabie A, Lee BT, Chen C, Lin SJ. Staged scalp soft tissue expansion before delayed allograft cranioplasty: a technical report. Neurosurgery 2012;71(1, Suppl Operative):15-20, discussion 21

8 Vogt PM, Mett TR, Broelsch GF, et al. Interdisciplinary reconstruction of oncological resections at the skull base, scalp and facial region. Surg Oncol 2017;26(03):318-323

9 Lin SJ, Hanasono MM, Skoracki RJ. Scalp and calvarial reconstruction. Semin Plast Surg 2008;22:281-293

10 Leedy JE, Janis JE, Rohrich RJ. Reconstruction of acquired scalp defects: an algorithmic approach. Plast Reconstr Surg 2005;116 (04):54e-72e

11 McCauley RL. Tissue expansion reconstruction of the scalp. Semin Plast Surg 2005;19(02):143-152

12 Keser A, Sensöz O, Mengi AS. Double opposing semicircular flap: a modification of opposing Z-plasty for closing circular defects. Plast Reconstr Surg 1998;102(04):1001-1007

13 Ahuja RB. Geometric considerations in the design of rotation flaps in the scalp and forehead region. Plast Reconstr Surg 1988;81(06): 900-906

14 Orticochea M. Four flap scalp reconstruction technique. Br J Plast Surg 1967;20(02):159-171

15 van Doormaal T, Kinaci A, van Thoor S, Redegeld S, Bergmann W, van der Zwan A. Usefulness of sealants for dural closure: evaluation in an in vitro model. Oper Neurosurg (Hagerstown) 2018;15 (04):425-432

16 Kinaci A, Algra A, Heuts S, O'Donnell D, van der Zwan A, van Doormaal T. Effectiveness of dural sealants in prevention of cerebrospinal fluid leakage after craniotomy: a systematic review. World Neurosurg 2018;118:368-376.e1

17 Hussussian CJ, Reece GP. Microsurgical scalp reconstruction in the patient with cancer. Plast Reconstr Surg 2002;109(06): 1828-1834

18 Raposio E, Nordström RE, Santi PL. Undermining of the scalp: quantitative effects. Plast Reconstr Surg 1998;101(05):1218-1222

19 Feroze AH, Walmsley GG, Choudhri O, Lorenz HP, Grant GA, Edwards MSB. Evolution of cranioplasty techniques in neurosurgery: historical review, pediatric considerations, and current trends. J Neurosurg 2015;123(04):1098-1107

20 Mehrara BJ, Disa JJ, Pusic A. Scalp reconstruction. J Surg Oncol 2006;94(06):504-508

21 Khan MN, Rodriguez LG, Pool CD, et al. The versatility of the serratus anterior free flap in head and neck reconstruction. Laryngoscope 2017;127(03):568-573

22 Pennington DG, Stern HS, Lee KK. Free-flap reconstruction of large defects of the scalp and calvarium. Plast Reconstr Surg 1989; 83(04):655-661

23 Aviv JE, Urken ML, Vickery C, Weinberg H, Buchbinder D, Biller HF. The combined latissimus dorsi-scapular free flap in head and neck 
reconstruction. Arch Otolaryngol Head Neck Surg 1991;117(11): $1242-1250$

24 Hwang JH, Lim SY, Pyon JK, Bang SI, Oh KS, Mun GH. Reliable harvesting of a large thoracodorsal artery perforator flap with emphasis on perforator number and spacing. Plast Reconstr Surg 2011;128(03):140e-150e

25 Yoshioka N. Versatility of the latissimus dorsi free flap during the treatment of complex postcraniotomy surgical site infections. Plast Reconstr Surg Glob Open 2017;5(06):e1355
26 Koolen PGL, Li Z, Roussakis E, et al. Oxygen-sensing paint-on bandage: calibration of a novel approach in tissue perfusion assessment. Plast Reconstr Surg 2017;140(01):89-96

27 Hosein RC, Cornejo A, Wang HT. Postoperative monitoring of free flap reconstruction: a comparison of external Doppler ultrasonography and the implantable Doppler probe. Plast Surg (Oakv) 2016;24(01):11-19

28 Keller A. Noninvasive tissue oximetry. Clin Plast Surg 2011;38 (02):313-324 